\title{
Exponential propagators for the Schrödinger equation with a time-dependent potential
}

Philipp Bader, Sergio Blanes, and Nikita Kopylov

Citation: The Journal of Chemical Physics 148, 244109 (2018); doi: 10.1063/1.5036838

View online: https://doi.org/10.1063/1.5036838

View Table of Contents: http://aip.scitation.org/toc/jcp/148/24

Published by the American Institute of Physics

\section{Articles you may be interested in}

Announcement: Top reviewers for The Journal of Chemical Physics 2017

The Journal of Chemical Physics 149, 010201 (2018); 10.1063/1.5043197

Inclusion of nuclear quantum effects for simulations of nonlinear spectroscopy

The Journal of Chemical Physics 148, 244105 (2018); 10.1063/1.5036768

Neural networks vs Gaussian process regression for representing potential energy surfaces: A comparative study of fit quality and vibrational spectrum accuracy

The Journal of Chemical Physics 148, 241702 (2018); 10.1063/1.5003074

Perspective: Multireference coupled cluster theories of dynamical electron correlation

The Journal of Chemical Physics 149, 030901 (2018); 10.1063/1.5039496

Investigating photoinduced proton coupled electron transfer reaction using quasi diabatic dynamics propagation

The Journal of Chemical Physics 148, 244102 (2018); 10.1063/1.5030634

Vibronic exciton theory of singlet fission. III. How vibronic coupling and thermodynamics promote rapid triplet generation in pentacene crystals

The Journal of Chemical Physics 148, 244701 (2018); 10.1063/1.5031778

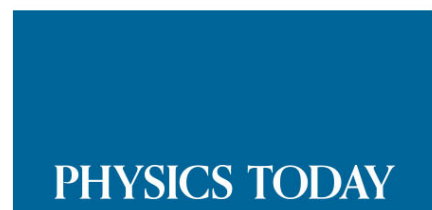

WHITEPAPERS
ADVANCED LIGHT CURE ADHESIVES

Take a closer look at what these environmentally friendly adhesive systems can do

\section{READ NOW}

PRESENTED BY

Q. MASTERBOND' 


\title{
Exponential propagators for the Schrödinger equation with a time-dependent potential
}

\author{
Philipp Bader, ${ }^{1, a)}$ Sergio Blanes, ${ }^{2, b)}$ and Nikita Kopylov ${ }^{2, c)}$ \\ ${ }^{1}$ Departament de Matemàtiques, Universitat Jaume I, E-12071 Castellón, Spain \\ ${ }^{2}$ Instituto Universitario de Matemática Multidisciplinar, Universitat Politècnica de València, \\ E-46022 Valencia, Spain
}

(Received 19 April 2018; accepted 11 June 2018; published online 28 June 2018)

\begin{abstract}
We consider the numerical integration of the Schrödinger equation with a time-dependent Hamiltonian given as the sum of the kinetic energy and a time-dependent potential. Commutator-free (CF) propagators are exponential propagators that have shown to be highly efficient for general time-dependent Hamiltonians. We propose new CF propagators that are tailored for Hamiltonians of the said structure, showing a considerably improved performance. We obtain new fourth- and sixth-order CF propagators as well as a novel sixth-order propagator that incorporates a double commutator that only depends on coordinates, so this term can be considered as cost-free. The algorithms require the computation of the action of exponentials on a vector similar to the well-known exponential midpoint propagator, and this is carried out using the Lanczos method. We illustrate the performance of the new methods on several numerical examples. Published by AIP Publishing. https://doi.org/10.1063/1.5036838
\end{abstract}

\section{INTRODUCTION}

We study the numerical integration of the time-dependent Schrödinger equation (SE) in units such that the Planck constant $\hbar=1$,

$$
i \frac{\partial}{\partial t} \psi(x, t)=\widehat{H}(t) \psi(x, t), \quad \psi\left(x, t_{0}\right)=\psi_{0}(x),
$$

where the Hamiltonian is given by

$$
\widehat{H}(t)=\widehat{T}+\widehat{V}(x, t)=-\frac{\Delta}{2 \mu}+\widehat{V}(x, t),
$$

where $x \in \mathbb{R}^{d}, \mu$ is the reduced mass, $\Delta$ is the Laplacian operator, and $\widehat{V}(x, t)$ is an explicitly time-dependent potential.

Although postponed spatial discretization has been proposed, ${ }^{1,2}$ the first step is usually discretizing Eq. (1) taking $N$ grid points. For this sake, various techniques can be used (see Ref. 3 and references therein). Thus, one obtains a linear ordinary differential equation

$$
i \frac{d}{d t} u(t)=H(t) u(t), \quad u\left(t_{0}\right)=u_{0} \in \mathbb{C}^{N},
$$

where $H(t)=T+V(t)$ is an $N \times N$ Hermitian matrix.

As in the existing literature, we assume the problem to be either periodic in space $x$ or to be considered as one due to solution $u(t)$ and its time-derivatives vanishing at the boundaries.

Thereafter, the time integration interval $\left[t_{0}, t_{f}\right]$ is divided in a number of sufficiently small time steps of length $\tau$, and stepwise approximations $u_{k}$ to the solution values $u\left(t_{k}\right)$

\footnotetext{
a)Electronic mail: bader@uji.es

b)Electronic mail: serblaza@imm.upv.es

c)Electronic mail: nikop1@upvnet.upv.es
}

are evaluated at the temporal grid points $t_{k}=t_{0}+\tau k$ $(k=1,2, \ldots)$.

Many algorithms for solving Eq. (3) exist indeed in the literature: split-operator methods, ${ }^{4}$ Runge-Kutta $(\mathrm{RK})^{5}$ and symplectic partitioned Runge-Kutta methods, ${ }^{6}$ a combination of a 4th-order Magnus method with the Lanczos algorithm, ${ }^{7}$ and the so-called $\left(t, t^{\prime}\right)$ method. ${ }^{8}$ In Refs. 7 and 9, a detailed analysis of these schemes was carried out.

There are two highly efficient families of propagators that have been adapted ${ }^{6,10-12}$ to the explicitly time-dependent case (3). Both make use of the fact that $V$ is diagonal in the coordinate space and fast Fourier transform (FFT) algorithms $\mathcal{F}$ can be used to diagonalize the kinetic energy operator $T=\mathcal{F}^{-1} T_{D} \mathcal{F}$.

In the first family, the solution is approximated by the unitary split operator algorithms, i.e., by compositions of the form

$$
\mathrm{e}^{-i \tau(T+V)} \approx \mathrm{e}^{-i b_{m} \tau V_{m}} \mathrm{e}^{-i a_{m} \tau T} \cdots c \mathrm{e}^{-i b_{1} \tau V_{1}} \mathrm{e}^{-i a_{1} \tau T}
$$

where $V_{j}=V\left(t_{k}+c_{j} \tau\right)$ and $\left\{a_{i}, b_{i}, c_{i}\right\}$ are appropriately chosen real coefficients. ${ }^{13-17}$ In this case, $m$ FFT calls (and their inverses, IFFT) per step are required. This class of methods shows high performance when $\|T\|,\|V\|,\|[T, V]\|$ $=\|T V-V T\|$ are relatively small or their action results have small norms, otherwise high errors can occur.

On the other hand, if $H(t)$ and its action $H(t) u(t)$ satisfy similar norm conditions as above, splitting $u(t)=q(t)+i p(t)$ into real and imaginary parts can be used. Equation (3) becomes

$$
\frac{d}{d t}\left(\begin{array}{l}
q \\
p
\end{array}\right)=\left[\left(\begin{array}{cc}
0 & H(t) \\
0 & 0
\end{array}\right)+\left(\begin{array}{cc}
0 & 0 \\
-H(t) & 0
\end{array}\right)\right]\left(\begin{array}{l}
q \\
p
\end{array}\right),
$$

that is similar to a set of $N$ coupled harmonic oscillators. Therefore, splitting methods tailored for that type of 
problems can be employed. $6,7,11,12,18$ The algorithm requires $2 m$ real-to-complex direct and inverse FFTs at a similar cost as $m$ complex-to-complex FFTs and is superior to the Chebyshev approximation for short and long time integrations. ${ }^{11}$ This class of methods is valid for any real Hamiltonian (not necessarily separable into kinetic and potential parts). These are symplectic methods that are conjugate to unitary ones. They are easy to apply and have low computational cost but are conditionally stable: if $\|H\|$ grows, one should reduce the time step for stability reasons.

In the present work, we consider commutator-free (CF) methods that address problems in which the above-mentioned techniques are not appropriate. Existing CF methods were, however, created for general Hamiltonians and do not take into account the specific structure (2).

The goal of this work is to construct fourth- and sixthorder geometric numerical integrators, which preserve most qualitative properties of the solution, ${ }^{13,19,20}$ by composition of maps $P_{m}$ that approximate

$$
u_{k+1}=\mathrm{e}^{-i \tau H} u_{k} \approx P_{m}(-i \tau H) u_{k},
$$

where $P_{m}$ is an approximation in the $m$-dimensional KrylovLanczos subspace. ${ }^{3,21,22}$

In essence, the three new methods utilize the 6th-order Gauss-Legendre (GL) quadrature nodes (but other quadrature rules can be used $\left.{ }^{10,30}\right)$,

$$
c_{1}=\frac{1}{2}-\frac{\sqrt{15}}{10}, \quad c_{2}=\frac{1}{2}, \quad c_{3}=\frac{1}{2}+\frac{\sqrt{15}}{10},
$$

to calculate linear combinations $\bar{V}_{i}=\sum_{j=1}^{3} a_{i, j} V_{j}$, with $V_{j}=V\left(t_{n}+c_{j} \tau\right)$ with $a_{i, j}$ specific to each method:

- the 4th-order method

$$
\Upsilon_{2}^{[4]}=\mathrm{e}^{-i \tau \bar{V}_{4}} \mathrm{e}^{-i \frac{\tau}{2}\left(T+\bar{V}_{3}\right)} \mathrm{e}^{-i \frac{\tau}{2}\left(T+\bar{V}_{2}\right)} \mathrm{e}^{-i \tau \bar{V}_{1}},
$$

- the 6th-order method that incorporates into ${ }^{8}$ one term, $\widetilde{V}$, containing derivatives of the potential

$$
\Upsilon_{2}^{[6]}=\mathrm{e}^{-i \tau\left(\bar{V}_{4}+\tau^{2} \widetilde{V}\right)} \mathrm{e}^{-i \frac{\tau}{2}\left(T+\bar{V}_{3}\right)} \mathrm{e}^{-i \frac{\tau}{2}\left(T+\bar{V}_{2}\right)} \mathrm{e}^{-i \tau\left(\bar{V}_{1}+\tau^{2} \widetilde{V}\right)},
$$

- the 6th-order one, with no derivatives of $V$,

$$
\Upsilon_{3}^{[6]}=\mathrm{e}^{-i \tau \bar{V}_{5}} \mathrm{e}^{-i \tau\left(a_{2} T+\bar{V}_{4}\right)} e^{-i \tau\left(a_{3} T+\bar{V}_{3}\right)} \mathrm{e}^{-i \tau\left(a_{2} T+\bar{V}_{2}\right)} \mathrm{e}^{-i \tau \bar{V}_{1}} .
$$

Since the first and last exponentials in the schemes (8)-(10) are diagonal matrices whose computational cost can be neglected, ${ }^{23}$ these methods require, in practice, the calculation of 2, 2, and 3 operator exponentials, respectively, which is done by the Krylov polynomial approximation (6).

The resulting schemes are 5/3 to 3 times more cost efficient than the existing CF methods, thanks to the simplified algebraic structure of the Hamiltonian written as the sum of a kinetic energy and a time-dependent potential and the low cost of exponentiating the latter.

In Sec. II, we describe the general methodology applied to obtaining commutator-free methods and existing specimens of this class and their limitations. In Sec. III, we perform manipulations that lead to explicit forms of new methods. The article concludes with numerical examples.

\section{METHODOLOGY}

\section{A. Commutator-free Magnus integrators}

For a sufficiently small time step, the solution of (3) can be expressed as the exponential of the Magnus expan$\operatorname{sion}^{24,25} \Omega(t)$. Computing the action of the $p$ th-order truncation, $\exp \Omega^{[p]}$, on a vector can be resource-consuming due to the commutators involved in $\Omega^{[p]}$.

To circumvent these overheads, one can approximate exponentials by a product of simpler matrices that do not contain commutators, e.g., a $r$-exponential method of order $p$ takes the form

$$
\mathrm{CF}_{r}^{[p]}=\prod_{j=1}^{r} \mathrm{e}^{-i \tau \widetilde{H}_{j}}=\mathrm{e}^{\Omega(\tau)}+\mathcal{O}\left(\tau^{p+1}\right),
$$

where

$$
\widetilde{H}_{j}=\sum_{l=1}^{m} a_{j, l} H\left(t_{k}+c_{l} \tau\right)=a_{j} T+\sum_{l=1}^{m} a_{j, l} V\left(t_{k}+c_{l} \tau\right)
$$

with $a_{j}=\sum_{l=1}^{m} a_{j, l}$. There exist highly efficient methods in the literature up to order eight. ${ }^{26-29}$ However, those methods are not optimized for problems in which the Hamiltonian is of form (2).

\section{B. Algebra and optimisation}

In this work, we use the 6th-order GL quadrature with nodes (7). Then, three linear combinations of $H\left(t_{k}+c_{j} \tau\right)=: T+V_{j}$,

$$
\begin{aligned}
& \alpha_{1}=-i \tau\left(T+V_{2}\right)=\mathcal{O}(\tau), \\
& \alpha_{2}=-i \tau \frac{\sqrt{15}}{3}\left(V_{3}-V_{1}\right)=\mathcal{O}\left(\tau^{2}\right), \\
& \alpha_{3}=-i \tau \frac{10}{3}\left(V_{3}-2 V_{2}+V_{1}\right)=\mathcal{O}\left(\tau^{3}\right),
\end{aligned}
$$

suffice $25,30,31$ to build methods up to order 6 .

Since $\alpha_{2}, \alpha_{3}$ are diagonal, and $\left[\alpha_{2}, \alpha_{3}\right]=0$, a 6thorder approximation $\Omega^{[6]}$ of the Magnus expansion $\Omega$ is given by

$\Omega^{[6]}=\alpha_{1}+\frac{1}{12} \alpha_{3}-\frac{1}{12}[12]+\frac{1}{360}[113]-\frac{1}{240}[212]+\frac{1}{720}[$

where $[i j \ldots k l]$ represents the nested commutator $\left[\alpha_{i},\left[\alpha_{j},[\ldots\right.\right.$, $\left.\left.\left.\left[\alpha_{k}, \alpha_{l}\right] \ldots\right]\right]\right]$. Condition $\left[\alpha_{2}, \alpha_{3}\right]=0$ makes that the coefficients need to satisfy a reduced number of order conditions to reach high orders.

Moreover, the operator [212] is also represented by a diagonal matrix whose elements are spatial derivatives of $\widehat{V}(x, t)$. This term can be combined with $\alpha_{2}$ and $\alpha_{3}$ to improve performance. Consequently, some $a_{j}$ can be zeroed and the computational cost (in terms of FFT calls) of corresponding exponentials in (11) can be neglected, as shown in Sec. III. 


\section{INTEGRATORS FOR THE SCHRÖDINGER EQUATION}

\section{A. Fourth-order methods}

An $m$-exponential method of order four that makes use of $\alpha_{1}, \alpha_{2}, \alpha_{3}$ given in (12) must satisfy

$$
\begin{aligned}
\Upsilon_{m}^{[4]} & =\prod_{i=1}^{m} \exp \left(x_{i, 1} \alpha_{1}+x_{i, 2} \alpha_{2}+x_{i, 3} \alpha_{3}\right) \\
& =\exp \left(\alpha_{1}+\frac{1}{12} \alpha_{3}-\frac{1}{12}[12]\right)+\mathcal{O}\left(\tau^{5}\right) .
\end{aligned}
$$

Time symmetry is preserved ${ }^{26}$ in a CF method when

$$
x_{m-i+1, j}=(-1)^{j+1} x_{i, j}, \quad i=1,2, \ldots
$$

We take $m=3, x_{1,1}=0$, and the result is the symmetric composition

$$
\begin{aligned}
\Upsilon_{3}^{[4]}= & \exp \left(-x_{1,2} \alpha_{2}+x_{1,3} \alpha_{3}\right) \exp \left(x_{2,1} \alpha_{1}+x_{2,3} \alpha_{3}\right) \\
& \times \exp \left(x_{1,2} \alpha_{2}+x_{1,3} \alpha_{3}\right)
\end{aligned}
$$

where the first and the last exponents are diagonal, and their computational cost is similar to a scheme with only one exponential, like the midpoint exponential method. This composition has 4 parameters to solve the order equations to assure 4 th order. Therefore, one free parameter remains for optimization. The scheme that satisfies the condition for [113] has the solution $^{32}$

$$
x_{1,2}=-\frac{1}{12}, \quad x_{1,3}=\frac{1}{60}, \quad x_{2,1}=1, \quad x_{2,3}=\frac{1}{20} .
$$

New 4th-order schemes with additional free parameters are given by composition

$$
\begin{aligned}
\Upsilon_{2}^{[4]}= & \exp \left(-x_{1,2} \alpha_{2}+x_{1,3} \alpha_{3}\right) \exp \left(x_{2,1} \alpha_{1}-x_{2,2} \alpha_{2}+x_{2,3} \alpha_{3}\right) \\
& \times \exp \left(x_{2,1} \alpha_{1}+x_{2,2} \alpha_{2}+x_{2,3} \alpha_{3}\right) \exp \left(x_{1,2} \alpha_{2}+x_{1,3} \alpha_{3}\right) .
\end{aligned}
$$

With two free parameters to satisfy the order conditions associated with [113] and [1112], we get the unique solution

$$
x_{1,2}=-x_{1,3}=-\frac{1}{60}, x_{2,1}=\frac{1}{2}, x_{2,2}=-\frac{2}{15}, x_{2,3}=\frac{1}{40} \text {. }
$$

Using (12), we can transform coefficients $x_{i, j}$ to $a_{i, j}$ as follows:

$$
a_{i, j}=\sum_{k=1}^{3} x_{i, k} G_{k, j}
$$

with

$$
G=\left(\begin{array}{ccc}
0 & 1 & 0 \\
-\frac{\sqrt{15}}{3} & 0 & \frac{\sqrt{15}}{3} \\
\frac{10}{3} & -\frac{20}{3} & \frac{10}{3}
\end{array}\right),
$$

and the method in terms of linear combinations of $T$ and $V_{j}$ becomes

$$
\Upsilon_{2}^{[4]}=\mathrm{e}^{-i \tau \bar{V}_{4}} \mathrm{e}^{-i \frac{\tau}{2}\left(T+\bar{V}_{3}\right)} \mathrm{e}^{-i \frac{\tau}{2}\left(T+\bar{V}_{2}\right)} \mathrm{e}^{-i \tau \bar{V}_{1}},
$$

where

$$
\begin{aligned}
& \bar{V}_{1}=a_{1,1} V_{1}+a_{1,2} V_{2}+a_{1,3} V_{3}, \\
& \bar{V}_{2}=a_{2,1} V_{1}+a_{2,2} V_{2}+a_{2,3} V_{3}, \\
& \bar{V}_{3}=a_{2,3} V_{1}+a_{2,2} V_{2}+a_{2,1} V_{3}, \\
& \bar{V}_{4}=a_{1,3} V_{1}+a_{1,2} V_{2}+a_{1,1} V_{3},
\end{aligned}
$$

and the coefficients are

$$
\begin{array}{lll}
a_{1,1}=\frac{10+\sqrt{15}}{180}, & a_{1,2}=-\frac{1}{9}, & a_{1,3}=\frac{10-\sqrt{15}}{180}, \\
a_{2,1}=\frac{15+8 \sqrt{15}}{90}, & a_{2,2}=\frac{2}{3}, & a_{2,3}=\frac{15-8 \sqrt{15}}{90} .
\end{array}
$$

All but one (i.e., [212]) order conditions at $\tau^{5}$ are satisfied in this symmetric method. The scheme can be written as

$$
\Upsilon_{2}^{[4]}=\exp \left(\Omega^{[6]}-z[212]+\mathcal{O}\left(\tau^{7}\right)\right), \quad z=\frac{1}{21600},
$$

that results in a highly optimized fourth-order method.

\section{B. Sixth-order methods}

Let us examine the operators

$$
\widehat{\alpha}_{1}=-i \tau\left(\widehat{T}+\widehat{V}_{2}(x)\right), \widehat{\alpha}_{2}=-i \tau \frac{\sqrt{15}}{3}\left(\widehat{V}_{3}(x)-\widehat{V}_{1}(x)\right) \text {. }
$$

The action of commutator $\left[\widehat{\alpha}_{2},\left[\widehat{\alpha}_{1}, \widehat{\alpha}_{2}\right]\right]$ can be expressed through spatial derivatives $\widehat{V}^{\prime}\left(x, t_{k}+c_{j} \tau\right) \equiv \widehat{V}_{j}^{\prime}$,

$$
[\widehat{212}] \psi(x, t)=i \tau^{3} \frac{5}{3 \mu}\left(\widehat{V}_{3}^{\prime}-\widehat{V}_{1}^{\prime}\right)^{2} \psi(x, t),
$$

and the corresponding matrix representation [212] is also diagonal in coordinate space. If spatial derivatives are relatively simple to evaluate, [212] can be used in (14) without significantly increasing its cost, similar to the high order force gradient algorithms in Refs. 33-35, yielding a 6th-order method given by

$$
\begin{aligned}
\Upsilon_{2}^{[6]}= & \exp \left(-x_{1,2} \alpha_{2}+x_{1,3} \alpha_{3}+y\left[\alpha_{2}, \alpha_{1}, \alpha_{2}\right]\right) \\
& \times \exp \left(x_{2,1} \alpha_{1}-x_{2,2} \alpha_{2}+x_{2,3} \alpha_{3}\right) \\
& \times \exp \left(x_{2,1} \alpha_{1}+x_{2,2} \alpha_{2}+x_{2,3} \alpha_{3}\right) \\
& \times \exp \left(x_{1,2} \alpha_{2}+x_{1,3} \alpha_{3}+y\left[\alpha_{2}, \alpha_{1}, \alpha_{2}\right]\right) .
\end{aligned}
$$

It is solved by the same $x_{i, j}$ as in (14), and

$$
y=\frac{z}{2}=\frac{1}{43200} \text {. }
$$

The coefficients of this method coincide with the scheme ${ }^{32}$ built for the numerical integration of Hill's equation.

With (12) and (17), (18) transforms to

$$
\Upsilon_{2}^{[6]}=\mathrm{e}^{-i \tau\left(\bar{V}_{4}+\tau^{2} \widetilde{V}\right)} \mathrm{e}^{-i \frac{\tau}{2}\left(T+\bar{V}_{3}\right)} \mathrm{e}^{-i \frac{\tau}{2}\left(T+\bar{V}_{2}\right)} \mathrm{e}^{-i \tau\left(\bar{V}_{1}+\tau^{2} \widetilde{V}\right)},
$$

and thanks to the diagonal form of $V(t)$,

$$
\widetilde{V}=-\frac{5 y}{3 \mu}\left(V^{\prime}\left(t_{k}+c_{3} \tau\right)-V^{\prime}\left(t_{k}+c_{1} \tau\right)\right)^{2} .
$$

Moreover, if $V(t)$ consists of a constant part $V_{(c)}$ and a time-dependent one, $V_{(t)}$, the modified potential $\widetilde{V}$ only requires spatial derivatives of $V_{(t)}$. In particular, for an external field problem $V_{(t)}=f(t) V_{(f)}$, one has 


$$
\widetilde{V} u_{k}=-\frac{1}{\mu} \frac{1}{25920}\left(f_{3}-f_{1}\right)^{2} V_{(f)}^{\prime 2} u_{k},
$$

with $f_{j}=f\left(t_{k}+c_{j} \tau\right)$ that is easily computed for predefined external interaction functions.

Nonetheless, in some cases $V^{\prime}(t)$ or an appropriate approximation can be difficult to obtain; hence, we consider schemes that have more exponentials but retain a relatively low computational cost.

Although 6th-order $\mathrm{CF}$ methods with four exponentials and no derivatives have been obtained, ${ }^{28}$ those schemes showed poor performance. Additional exponentials have to be incorporated into the scheme to improve its performance. ${ }^{26,28}$ We insert an exponential in the middle, and the composition contains 2 free parameters. To reduce computational cost, we use one parameter to eliminate contributions from $\alpha_{1}$ in the outermost exponentials, $x_{1,1}=0$. We note that minimizing $\sum_{i, j}\left|x_{i, j}\right|$ or $\sum_{i, j}\left|x_{i, j}\right|^{2}$ leads to virtually identical performance; hence, we have opted for simplicity. There is one free parameter among $x_{1,3}, x_{2,3}$, and $x_{3,3}$ that multiplies $\alpha_{3}$, and they appear only in two of the order conditions. Thus, we set $x_{1,3}=0$ to simplify the exponential further,

$$
\begin{aligned}
\Upsilon_{3}^{[6]}= & \exp \left(-x_{1,2} \alpha_{2}\right) \times \exp \left(x_{2,1} \alpha_{1}-x_{2,2} \alpha_{2}+x_{2,3} \alpha_{3}\right) \\
& \times \exp \left(x_{3,1} \alpha_{1}+x_{3,3} \alpha_{3}\right) \exp \left(x_{2,1} \alpha_{1}+x_{2,2} \alpha_{2}+x_{2,3} \alpha_{3}\right) \\
& \times \exp \left(x_{1,2} \alpha_{2}\right) \\
= & \mathrm{e}^{-i \tau \bar{V}_{5}} \mathrm{e}^{-i \tau\left(a_{2} T+\bar{V}_{4}\right)} e^{-i \tau\left(a_{3} T+\bar{V}_{3}\right)} \mathrm{e}^{-i \tau\left(a_{2} T+\bar{V}_{2}\right)} \mathrm{e}^{-i \tau \bar{V}_{1}}
\end{aligned}
$$

where

$$
\begin{aligned}
& \bar{V}_{1}=a_{1,1} V_{1}+a_{1,2} V_{2}+a_{1,3} V_{3}, \\
& \bar{V}_{2}=a_{2,1} V_{1}+a_{2,2} V_{2}+a_{2,3} V_{3}, \\
& \bar{V}_{3}=a_{3,1} V_{1}+a_{3,2} V_{2}+a_{3,3} V_{3}, \\
& \bar{V}_{4}=a_{2,3} V_{1}+a_{2,2} V_{2}+a_{2,1} V_{3}, \\
& \bar{V}_{5}=a_{1,3} V_{1}+a_{1,2} V_{2}+a_{1,1} V_{3},
\end{aligned}
$$

and we choose the only real valued solution that gives

$$
\begin{aligned}
a_{1,1} & =0.01994096265093610745, \\
a_{1,2} & =0 \\
a_{1,3} & =-a_{1,1}, \\
a_{2,1} & =0.4882524910228221957, \\
a_{2,2} & =-0.0046136830175630621, \\
a_{2,3} & =0.0834019108602182940 \\
a_{3,1} & =-0.29387662410526271191, \\
a_{3,2} & =0.4536718104795705687, \\
a_{3,3} & =a_{3,1}, \\
a_{2} & =\sum_{i=1}^{3} a_{2, i}=0.56704071886547742757, \\
a_{3} & =\sum_{i=1}^{3} a_{3, i}=1-2 a_{2} \\
& =-0.13408143773095485515 .
\end{aligned}
$$

The analysis of this family of methods carried out in Ref. 36 shows that there exist no 6th-order commutatorfree methods with all coefficients $x_{i, 1}=a_{i}$ greater than zero.

\section{NUMERICAL ILLUSTRATIONS}

In this section, we illustrate the methods' behavior and performance on academic one-dimensional examples.

First, let us describe the general setup used for numerical experiments in this paper. We define the wave function $\psi(x$, $t)$ on a sufficiently large spatial domain $\left[x_{0}, x_{N}\right)$ to ensure that its value and its derivatives vanish. This allows us to impose periodic boundary conditions $\psi\left(x_{0}, t\right)=\psi\left(x_{N}, t\right)$ and hence the use of FFTs. We divide the interval into $N$ bins of length $\Delta x=\left(x_{N}-x_{0}\right) / N$; hence, $x_{k}=x_{0}+k \Delta x, k=0, \ldots$, $N-1$. The discrete vector $u(t) \in \mathbb{C}^{N}$ from (3) has components $u_{k}=(\Delta x)^{1 / 2} \psi\left(x_{k}, t\right)$, and its norm $\|u(t)\|_{2}$ does not depend on $t$.

Under these assumptions, we consider the onedimensional Schrödinger equation $(\hbar=1)$,

$$
\begin{aligned}
i \frac{\partial}{\partial t} \psi(x, t) & =\left(-\frac{1}{2 \mu} \frac{\partial^{2}}{\partial x^{2}}+V(x)+f(t) x\right) \psi(x, t), \\
\psi(x, 0) & =\psi_{0}(x) .
\end{aligned}
$$

The time-dependent part $f(t)=A \cos (\omega t)$ corresponds to an external laser field.

To check accuracy, we calculate the reference solution at the final time $u\left(t_{f}\right)$ using a sufficiently small time step. Then, we compute the solution with each method for various numbers of time steps and measure the 2-norm of the errors at the final time. The cost of a scheme is counted in units of complex-tocomplex FFT-IFFT pairs required for calculating exponentials in each step.

In all the examples, exponentials are approximated by the Lanczos method according to the procedure described in Appendix A.

Finally, we plot the errors for each time step versus the corresponding cost in the double logarithmic scale.

In our experiments, we compare with the commutator-free methods obtained in Ref. 28: the 4th-order method with 3 exponentials (CF4:3Opt in the source notation, $\mathrm{CF}_{3 O p t}^{[4]}$ here) and the 6th-order scheme with five exponentials (CF6:5 =: $\mathrm{CF}_{5}^{[6]}$ ). The 6th-order scheme with six exponentials (CF6:6) showed worst performance in our numerical experiments and it has not been considered. Although in Ref. 28 the authors present other optimized 6th-order methods that use the nodes of the 8th-order GL quadrature rule, our schemes can also use higherorder quadrature rules (as shown in Appendix B), and for this reason, we limit ourselves to the comparison of propagators that are similarly based on the three-point GL rule. Thus, each exponential is written in terms of the linear combinations of $H_{i}=H\left(t_{k}+c_{i} \tau\right)$ with $c_{i}, i=1,2,3$ given in (7), 


$$
\begin{aligned}
\mathrm{CF}_{5}^{[6]} & =\prod_{i=1}^{5} \mathrm{e}^{-i \tau\left(a_{i, 1} H_{1}+a_{i, 2} H_{2}+a_{i, 3} H_{3}\right)}, \\
a_{1,1} & =0.203952578716323, a_{1,2}=-0.059581898090478, a_{1,3}=0.015629319374155, \\
a_{2,1} & =0.133906069544898, a_{2,2}=0.314511533222506, a_{2,3}=-0.060893550742092, \\
a_{3,1} & =-0.014816639115506, a_{3,2}=-0.065414825819611, a_{3,3}=-0.014816639115506, \\
a_{i, j} & =a_{6-i, 4-j}, i=4,5, j=1,2,3 .
\end{aligned}
$$

We also consider the Magnus-based exponential methods where a second-order method is given by the first term in the expansion

$$
\Upsilon^{[2]}=\exp \left(-i \int_{t_{k}}^{t_{k}+\tau} H(t) d t\right)
$$

that provides a second-order approximation to the solution. Then, it is sufficient to approximate the integral by the midpoint quadrature rule of order two,

$$
\Upsilon_{1}^{[2]}=\mathrm{e}^{-i \tau\left(T+V\left(t_{k}+\tau / 2\right)\right)} \approx P_{m}\left(-i \tau\left(T+V_{\frac{\tau}{2}}\right)\right) .
$$

However, as it is the case for all the new methods presented in this work, the schemes can be used using higher-order quadrature rules. For example, we can approximate the integral using the 6th-order GL rule (7) as for all methods tested in this work, so the use of the following averaged one-exponential method is considered:

$$
\Upsilon_{1,3}^{[2]}=\mathrm{e}^{\alpha_{1}+\frac{1}{12} \alpha_{3}}=\mathrm{e}^{-i \tau\left(T+\frac{1}{18}\left(5 V_{1}+8 V_{2}+5 V_{3}\right)\right)}
$$

with $V_{i}=V\left(t_{k}+c_{i} \tau\right)$. It is worth noting that if the integral approximation contains the dominant error of the method, $\Upsilon_{1,3}^{[2]}$ should provide more accurate result without increasing the computational cost.

We also consider the 4th-order enhanced one-exponential Magnus

$$
\Upsilon_{1}^{[4]}=\mathrm{e}^{\Omega^{[4]}}=\mathrm{e}^{\alpha_{1}+\frac{1}{12} \alpha_{3}-\frac{1}{12}\left[\alpha_{1}, \alpha_{2}\right]},
$$

where the evaluation of $\Omega^{[4]} u$ requires two FFT-IFFT transforms.

The salient point is that the new $\Upsilon_{2}^{[4]}$ and $\Upsilon_{2}^{[6]}$ are minor modifications of two consecutive steps of (28) and, in most cases, their overall cost is similar because the higher-order methods can be used with a time step twice larger.

The Walker-Preston model is a simple model that represents adequately many typical applications and may serve as an indicative benchmark.

We take the Morse potential $V(x)=D\left(1-\mathrm{e}^{-\alpha x}\right)^{2}$ for $x \in[-0.8,4.32)$, subdivided into $N=64$ and $N=128$. The parameters chosen are as follows: $\mu=1745$ a.u., $D=0.2251$ a.u., and $\alpha=1.1741$ a.u.. The amplitude is $A=A_{0}=0.011025$ a.u. and the frequency is $\omega=\omega_{0}=0.01787$. This corresponds to a standard example of the diatomic HF molecule in a strong laser field. ${ }^{37}$

The numerical experiments are repeated with reduced intensity and frequency $A=A_{0} / 2$ and $\omega=\omega_{0} / 2$, leaving time steps the same.
As an initial condition, we take the ground state of the Morse potential

$$
\phi(x)=\sigma \exp \left(-\left(\gamma-\frac{1}{2}\right) \alpha x\right) \exp \left(-\gamma \mathrm{e}^{-\alpha x}\right),
$$

with $\gamma=2 D / w_{0}, w_{0}=\alpha \sqrt{2 D / \mu}$, and $\sigma$ is a normalizing constant. We integrate on $\left[0,10 t_{p}\right]$ with $t_{p}=2 \pi / \omega$.

Figure 1 shows the efficiency plots (the two-norm error versus the number of FFT calls in the logarithmic scale) of

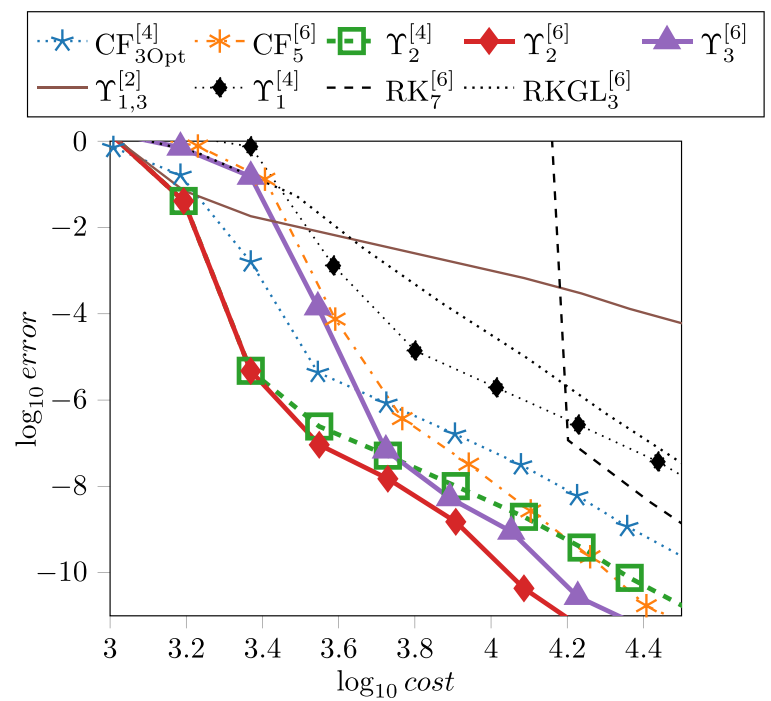

(a)

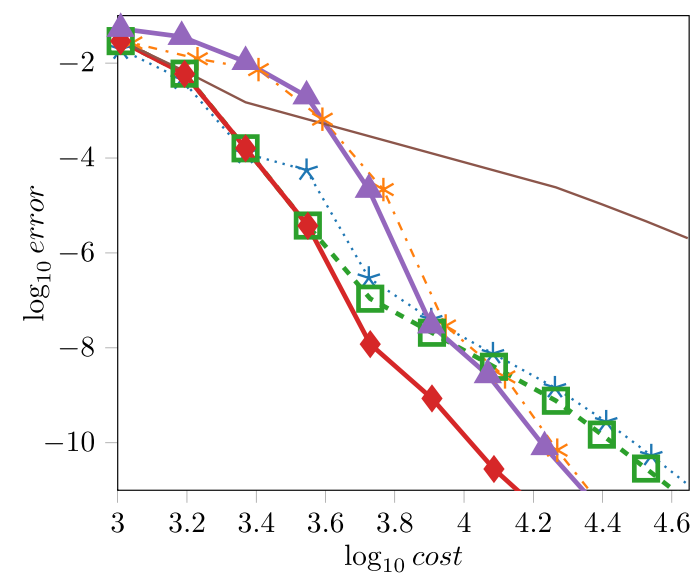

(b)

FIG. 1. Efficiency plots of the methods described below for the WalkerPreston model, $N=64$. Schemes $\Upsilon_{1}^{[4]}, \mathrm{RK}_{7}^{[6]}$, RKGL ${ }_{3}^{[6]}$ are reference methods, and they are only plotted in the top figure due to their inferior performance. (a) $\omega=\omega_{0}, A=A_{0}$ (b) $\omega=\omega_{0} / 2, A=A_{0} / 2$. 
the 4th- and 6th-order methods for the Walker-Preston model with $N=64$ for the following methods:

- $\mathrm{CF}_{3 O p t}^{[4]}, \mathrm{CF}_{5}^{[6]}$ : the 3- and 5-exponential CF methods of order four and six from Ref. 28.

- the enhanced lower-order Magnus methods: midpoint $\Upsilon_{1,3}^{[2]}(28)$; the 4th-order $\Upsilon_{1}^{[4]}(29)$.

- the new methods: $\Upsilon_{2}^{[4]}$ given by (16) and (17); $\Upsilon_{2}^{[6]}$ given by (19); $\Upsilon_{3}^{[6]}$ given by (20)-(23).

- the classical 6th-order methods: an explicit 7-stage $\mathrm{RK}_{7}^{[6]}$ method and the implicit symplectic $\mathrm{RKGL}_{3}^{[6]}$ method.

The same experiments are repeated for $N=128$, and the results are shown in Fig. 2.

From Fig. 1 (top), it is evident that the classical RK methods have inferior performance (moreover, the explicit RK requires small time steps for stability) and are omitted from

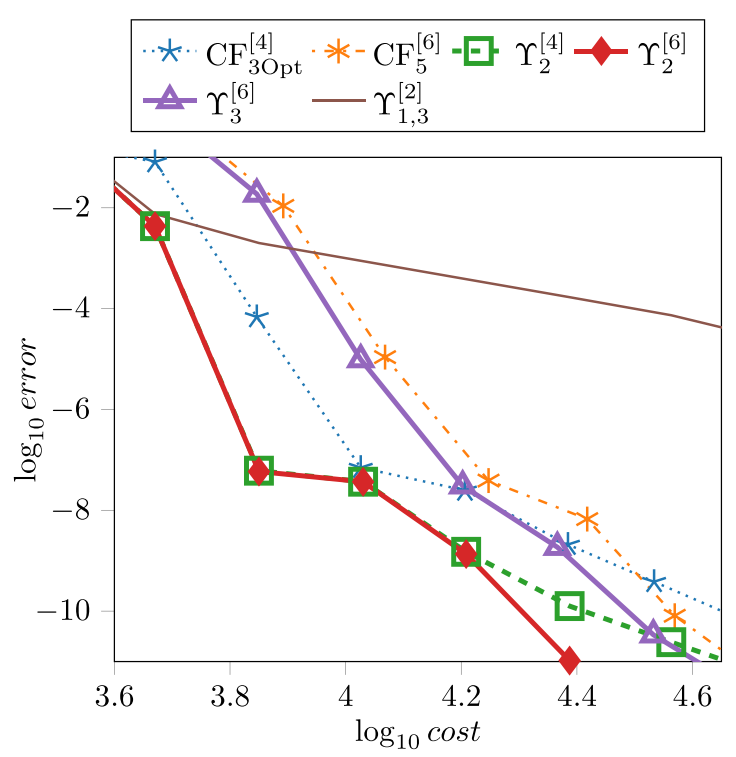

(a)

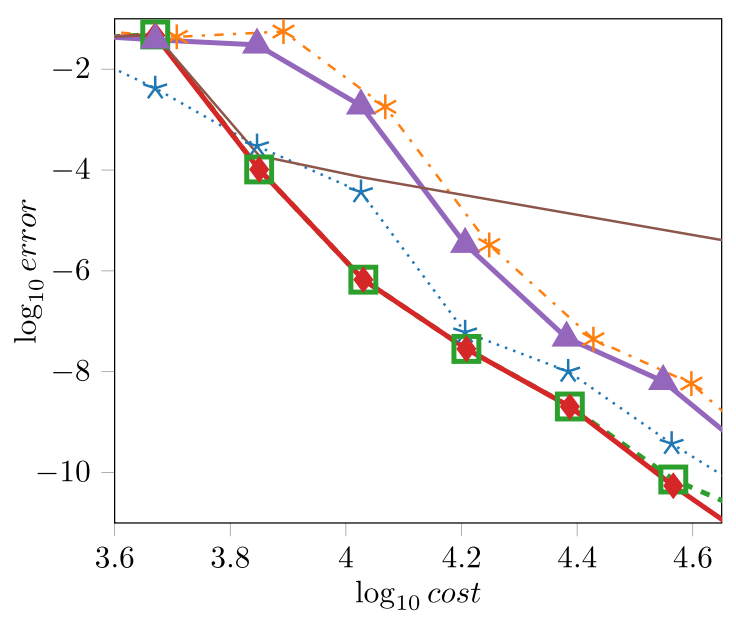

(b)

FIG. 2. Efficiency plots for the Walker-Preston model, $N=128 . \Upsilon_{1}^{[4]}, \mathrm{RK}_{7}^{[6]}$, $\mathrm{RKGL}_{3}^{[6]}$ are omitted for clarity. (a) $\omega=\omega_{0}, A=A_{0}$ (b) $\omega=\omega_{0} / 2$, $A=A_{0} / 2$. the other plots. We observe that both new 6th-order methods perform best when high accuracy is desired. The 4th-order $\Upsilon_{2}^{[4]}$ demonstrates comparable performance when large time steps are used and do not require the evaluation of the spatial derivative of the potential. We observe that the new methods are better than the exponential midpoint for all accuracies of practical interest and have similar complexity for their implementation; so, any user of the exponential midpoint method should consider one of the new methods for solving this class of problems.

\section{CONCLUSION}

We have constructed methods for the time-dependent Hamiltonian $H(t)$ that can be presented as the sum of the kinetic energy $\widehat{T}$ and an explicitly time-dependent potential $\widehat{V}(t)$. The obtained methods belong to the class of so-called commutatorfree (quasi-Magnus) integrators and heavily rely on the properties of the problem, hence their reduced computational cost.

On widely used benchmark problems, we have shown appropriateness of using the new methods to solve (1). All new schemes have shown better performance than the noncustomized methods of the same family. Summarizing, $\Upsilon_{2}^{[6]}$ is the method of choice when spatial derivatives of $\widehat{V}(x, t)$ are available, and $\Upsilon_{3}^{[6]}$ can be used otherwise. The 4th-order $\Upsilon_{2}^{[4]}$ is appropriate for use with larger time steps when high accuracy is not required. In addition, these methods have shown to be superior to the exponential midpoint for all desired accuracies of practical interest and have similar complexity for their implementation.

Future work may concern constructing of methods of higher order that employ other quadrature rules and can compete with 8 th-order $\mathrm{CF}$ methods ${ }^{28}$ or the recent methods with simplified commutators considered in Ref. 38. Moreover, it seems possible to apply the same technique to tailoring methods for other problems with the special structure which may allow additional optimization.

\section{ACKNOWLEDGMENTS}

We wish to acknowledge Fernando Casas for his help in the construction of the methods $\Upsilon_{3}^{[6]}$. The authors acknowledge Ministerio de Economía y Competitividad (Spain) for financial support through Project No. MTM2016-77660-P (AEI/FEDER, UE). Additionally, Kopylov has been partly supported by Grant No. GRISOLIA/2015/A/137 from the Generalitat Valenciana.

\section{APPENDIX A: KRYLOV APPROXIMATION WITH LANCZOS METHOD}

If $H$ is Hermitian and $u$ is a unit vector, then $\mathrm{e}^{-i \tau H} u_{k}$ can be approximated in the $m$-dimensional Krylov subspace, ${ }^{21}$ whose column basis $V_{m}=\left\{v_{1}, v_{2}, \ldots, v_{m}\right\}:=\left\{u_{k}, H u_{k}, \ldots, H^{m-1} u_{k}\right\}$ is constructed by the Lanczos algorithm. ${ }^{3}$ Exponentiation of the full operator is reduced to the exponentiation of a tridiagonal symmetric matrix $T_{m}$ of smaller 
dimension

$$
\mathrm{e}^{-i \tau H} u_{k} \approx V_{m} \mathrm{e}^{-i \tau T_{m}} e_{1},
$$

where $e_{1}$ is the first column of the identity matrix.

The Lanczos algorithm builds an orthogonal basis and fills elements of $T_{m},{ }^{3}$

$$
\begin{aligned}
& v_{0}=0, \quad v_{1}=u_{k}, \quad \beta_{1}=0 \\
& \text { for } i=1, \ldots, m \\
& \quad y=\tau H v_{i}-\beta_{i} v_{i-1} \\
& \quad \alpha_{i}=\left\langle v_{i} \mid y\right\rangle \\
& \quad y=y-\alpha_{i} v_{i} \\
& \quad \beta_{i+1}=\|y\| \\
& \quad v_{i+1}=y / \beta_{i+1} \\
& \text { endfor } \\
& \alpha_{i}=\left(T_{m}\right)_{i, i}, \beta_{i+1}=\left(T_{m}\right)_{i+1, i}, i=1, \ldots, m-1 .
\end{aligned}
$$

The procedure requires $m$ matrix-vector products, storing $m$ resulting vectors, and at the end discarding $v_{m+1}$ and $\beta_{m+1}$ to produce square matrices.

The error can be cheaply estimated by

$$
e r r=\beta_{m+1}\left(\frac{2}{3}\left|e_{m}^{T} e^{-i \tau T_{m} / 2} e_{1}\right|+\frac{1}{6}\left|e_{m}^{T} e^{-i \tau T_{m}} e_{1}\right|\right),
$$

where $e_{1}, e_{m}$ are the first and last columns of the $m \times m$ identity matrix.

Thus, in this work, the procedure is the following: given a tolerance tol, apply the Lanczos algorithm to build the sequence of vectors $\left\{u_{k}, H u_{k}, H^{2} u_{k}, \ldots\right\}$ and fill $T_{m}$ until err $<$ tol or $m=10$ is reached to ensure a sufficiently small error for a 6 th-order method. Having obtained $V_{m}$ and $T_{m}$, then (A1) is computed.

\section{APPENDIX B: SCHEMES WITH NODES OF AN ARBITRARY QUADRATURE RULE}

The methods proposed in this work can be used with any other quadrature rule of order $r \geq 6$, say $\left\{\tilde{b}_{i}, \tilde{c}_{i}\right\}_{i=1}^{k}$, where $\tilde{b}_{i}$ are the weights and $\tilde{c}_{i}$ are the nodes. Similarly as in Ref. 10, and as explicitly shown in Ref. 30 , there is a simple rule to apply: to replace $\alpha_{1}, \alpha_{2}, \alpha_{3}$ in (12) by

$$
\left(\begin{array}{c}
\alpha_{1} \\
\alpha_{2} \\
\alpha_{3}
\end{array}\right)=G Q^{-1} \tilde{Q}\left(\begin{array}{c}
\tilde{H}_{1} \\
\vdots \\
\tilde{H}_{k}
\end{array}\right)
$$

with $\tilde{H}_{i}=H\left(t+\tilde{c}_{i} \tau\right), i=1, \ldots, k, G$ given by $(15)$ and

$$
Q_{i, j}=b_{j}\left(c_{j}-\frac{1}{2}\right)^{i-1}, \quad \tilde{Q}_{i, l}=\tilde{b}_{l}\left(\tilde{c}_{k}-\frac{1}{2}\right)^{i-1},
$$

where $i, j=1,2,3, l=1, \ldots, k$, with $\left(b_{1}, b_{2}, b_{3}\right)=\frac{1}{18}(5,8,5)$ being the weights of the 6th-order GL quadrature rule with nodes $c_{i}$ given in (7).

Obviously, the element [212] to be used in the scheme $\Upsilon_{2}^{[6]}$ remains diagonal but now in terms the spatial derivatives of the potential evaluated at the new quadrature nodes.

${ }^{1}$ P. Bader, A. Iserles, K. Kropielnicka, and P. Singh, Found. Comput. Math. 14, 689 (2014).

${ }^{2}$ P. Bader, A. Iserles, K. Kropielnicka, and P. Singh, Proc. R. Soc. A 472(2193), 20150733 (2016).

${ }^{3}$ C. Lubich, From Quantum To Classical Molecular Dynamics: Reduced Models and Numerical Analysis (European Mathematical Society, 2008).

${ }^{4}$ M. D. Feit, J. A. Fleck, Jr., and A. Steiger, J. Comput. Phys. 47, 412 (1982).

${ }^{5}$ J. Tremblay and T. Carrington, Jr., J. Chem. Phys. 121, 11535 (2004).

${ }^{6}$ J. Sanz-Serna and A. Portillo, J. Chem. Phys. 104, 2349 (1996).

${ }^{7}$ K. Kormann, S. Holmgren, and H. Karlsson, J. Chem. Phys. 128, 184101 (2008).

${ }^{8}$ U. Peskin, R. Kosloff, and N. Moiseyev, J. Chem. Phys. 100, 8849 (1994).

${ }^{9}$ A. Castro, M. Marques, and A. Rubio, J. Chem. Phys. 121, 3425 (2004).

${ }^{10}$ S. Blanes, F. Casas, and A. Murua, J. Chem. Phys. 146, 114109 (2017).

${ }^{11}$ S. Blanes, F. Casas, and A. Murua, J. Comput. Phys. 303, 396 (2015).

${ }^{12}$ S. Gray and J. Verosky, J. Chem. Phys. 100, 5011 (1994).

${ }^{13} \mathrm{~S}$. Blanes and F. Casas, A Concise Introduction to Geometric Numerical Integration, Monographs and Research Notes in Mathematics (CRC Press, 2016).

${ }^{14}$ R. I. McLachlan and R. Quispel, Acta Numer. 11, 341 (2002).

${ }^{15}$ C. Neuhauser and M. Thalhammer, BIT Numer. Math. 49, 199 (2009).

${ }^{16}$ M. Thalhammer, SIAM J. Numer. Anal. 46, 2022 (2008).

${ }^{17}$ M. Thalhammer, SIAM J. Numer. Anal. 50, 3231 (2012).

${ }^{18}$ S. Gray and D. Manolopoulos, J. Chem. Phys. 104, 7099 (1996).

${ }^{19}$ E. Hairer, C. Lubich, and G. Wanner, Geometric Numerical Integration. Structure-Preserving Algorithms for Ordinary Differential Equations, 2nd ed. (Springer-Verlag, 2006).

${ }^{20}$ J. M. Sanz-Serna and M. P. Calvo, Numerical Hamiltonian Problems, AMMC 7 (Chapman \& Hall, 1994).

${ }^{21}$ Y. Saad, SIAM J. Numer. Anal. 29, 209 (1992).

${ }^{22}$ T. Park and J. Light, J. Chem. Phys. 85, 5870 (1986).

${ }^{23}$ S. Blanes and P. C. Moan, Phys. Lett. A 265, 35 (2000).

${ }^{24}$ W. Magnus, Commun. Pure Appl. Math. 7, 649 (1954).

${ }^{25}$ S. Blanes, F. Casas, J. A. Oteo, and J. Ros, Phys. Rep. 470, 151 (2009).

${ }^{26}$ S. Blanes and P. C. Moan, Appl. Numer. Math. 56, 1519 (2006).

${ }^{27}$ M. Thalhammer, SIAM J. Numer. Anal. 44, 851 (2006).

${ }^{28}$ A. Alvermann and H. Fehske, J. Comput. Phys. 230, 5930 (2011).

${ }^{29}$ N. Auer, L. Einkemmer, P. Kandolf, and A. Ostermann, Comput. Phys. Commun. 228, 115 (2018).

${ }^{30} \mathrm{~S}$. Blanes, "Time-average on the numerical integration of non-autonomous differential equations," SIAM J. Numer. Anal (to be published).

${ }^{31}$ H. Munthe-Kaas and B. Owren, Philos. Trans. R. Soc., A 357, 957 (1999).

${ }^{32}$ P. Bader, S. Blanes, E. Ponsoda, and M. Seydaoğlu, J. Comput. Appl. Math. 316, 47 (2017).

${ }^{33}$ P.-V. Koseleff, "Formal calculus for Lie methods in Hamiltonian mechanics," Ph.D. thesis, Lawrence Berkeley Laboratory, 1994.

${ }^{34}$ S. A. Chin, Phys. Lett. A 226, 344 (1997).

${ }^{35}$ I. P. Omelyan, I. M. Mryglod, and R. Folk, Phys. Rev. E 66, 026701 (2002).

${ }^{36}$ H. Hofstätter and O. Koch, e-print arXiv: 1709.08473.

${ }^{37}$ R. Walker and K. Preston, J. Chem. Phys. 67, 2017 (1977).

${ }^{38}$ A. Iserles, K. Kropielnicka, and P. Singh, SIAM J. Num. Anal. 56, 15471569 (2018). 